\title{
NOTES \\ ON THE NUMERICAL ACCURACY OF FIRST-ORDER APPROXIMATE SOLUTIONS TO DSGE MODELS
}

\author{
Christopher Heiberger, Torben Klarl, and Alfred Maussner \\ University of Augsburg
}

\begin{abstract}
Many algorithms that provide approximate solutions for dynamic stochastic general equilibrium (DSGE) models employ the QZ factorization because it allows a flexible formulation of the model and exempts the researcher from identifying equations that give raise to infinite eigenvalues. We show, by means of an example, that the policy functions obtained by this approach may differ from both the solution of a properly reduced system and the solution obtained from solving the system of nonlinear equations that arises from applying the implicit function theorem to the model's equilibrium conditions. As a consequence, simulation results may depend on the specific algorithm used and on the numerical values of parameters that are theoretically irrelevant. The sources of this inaccuracy are ill-conditioned matrices as they emerge, e.g., in models with strong habits. Researchers should be aware of those strange effects, and we propose several ways to handle them.
\end{abstract}

Keywords: DSGE Models, QZ Factorization, System Reduction, Accuracy of Solutions

\section{INTRODUCTION}

Dynamic stochastic general equilibrium (DSGE) models have become the workhorse of macroeconomic research. Although the early proponents of this approach had to write their own computer code, their contemporary successors can resort to a variety of freely available toolkits, among which DYNARE is probably the best-known and most versatile. ${ }^{1}$ The user-friendly toolkits have spurred the further development and prevalence of DSGE models, because they have reduced the barriers for potential users considerably. One does not have to understand the details of a particular algorithm, the pitfalls of numerical mathematics, or the subtleties of different programming languages to solve, simulate, and even estimate a particular model.

\footnotetext{
This paper is a substantially revised and extended version of our former working paper entitled "System Reduction and the Accuracy of Solutions of DSGE Models: A Note." We are grateful to two anonymous referees for their comments and suggestions. Of course, all remaining errors and shortcomings are ours. Alfred Maußner acknowledges financial support by the Deutsche Forschungsgemeinschaft within the priority program "Financial Market Imperfections and Macroeconomic Performance" under Grant MA 1110/3-1. Address correspondence to: Alfred Maußner, Department of Economics, University of Augsburg, Universitätsstraße 16, 86159 Augsburg, Germany; e-mail: alfred.maussner@wiwi.uni-augsburg.de.
} 
In this paper we argue for careful use. In particular, we illustrate by means of an example different degrees of numerical accuracy that depend on the particular algorithm used to obtain the linear part of the model's approximate solution. Errors that occur at this stage affect the computation of higher-order terms of the solution. The example is by no means specific. Rather, versions of this model are routinely employed in studies of the equity premium puzzle. ${ }^{2}$

Our benchmark is the (stable) solution of the system of nonlinear equations obtained from applying the implicit function theorem to the model's equilibrium conditions. We compare this solution with those that result from linearizing the equilibrium conditions and from solving the respective stochastic first-order system of difference equations. The prevalent way to do this is to use matrix factorization. We consider QZ factorization and, for a properly reduced system, Schur decomposition. Theoretically, i.e., ignoring errors from finite-precision computer arithmetic, all these algorithms will deliver the same solution. In our application, however, some of the elements of the solution differ remarkably between the various methods. As a consequence, we also observe differences in the second moments of simulated time series.

The researcher who relies on the use of DSGE toolkits thus should be aware of these strange effects. We propose several ways to handle them. Euler equation residuals, error bounds for the eigenvalues, and the nonlinear equations can be used to detect a potential problem. A reformulation of the model in terms of transformed variables or equations and a specific balancing of ill-conditioned matrices are ways to improve accuracy.

From here we proceed with a brief description of the canonical DSGE model, the linearized form of this model, and the matrix factorizations in the next section. Section 3 presents our example. Section 4 concludes. The Online Appendix covers the details of the matrix factorizations and of the model presented in Section 3 and provides additional information on the model's approximate solutions. ${ }^{3}$

\section{ANALYTICAL FRAMEWORK}

\subsection{Canonical Dynamic Stochastic General Equilibrium Model}

Our framework closely follows Schmitt-Grohé and Uribe (2004). The solution based on the QZ factorization is due to Klein (2000) and the presentation follows Heiberger et al. (2015).

Let $\mathbf{x}_{t} \in \mathbf{R}^{n(x)}, \mathbf{z}_{t} \in \mathbf{R}^{n(z)}$, and $\mathbf{y}_{t} \in \mathbf{R}^{n(y)}$ denote a vector of endogenous state variables, exogenous state variables, and nonpredetermined (jump) variables, respectively. The equilibrium conditions of a DSGE model are

$$
\begin{aligned}
\mathbf{0}_{n(x)+n(y)} & =\mathbf{E}_{t} \mathbf{g}\left(\mathbf{x}_{t}, \mathbf{z}_{t}, \mathbf{y}_{t}, \mathbf{x}_{t+1}, \mathbf{z}_{t+1}, \mathbf{y}_{t+1}\right), \\
\mathbf{z}_{t+1} & =\Pi \mathbf{z}_{t}+\sigma \Omega \boldsymbol{\epsilon}_{t+1}, \quad \boldsymbol{\epsilon}_{t+1} \sim \mathcal{N}\left(\mathbf{0}_{n(z)}, I_{n(z)}\right),
\end{aligned}
$$


where the operator $\mathbf{E}_{t}$ denotes expectations as of period $t$. Perturbation methods yield approximate solutions

$$
\begin{aligned}
\mathbf{x}_{t+1} & =\mathbf{h}^{x}\left(\mathbf{x}_{t}, \mathbf{z}_{t}, \sigma\right), \\
\mathbf{y}_{t} & =\mathbf{h}^{y}\left(\mathbf{x}_{t}, \mathbf{z}_{t}, \sigma\right) .
\end{aligned}
$$

\subsection{The Set of Nonlinear Equations}

Let $\mathbf{w}_{t}=\left[\mathbf{x}_{t}^{\prime}, \mathbf{z}_{t}^{\prime}\right]^{\prime}, \tilde{\Omega}=\left[\mathbf{0}_{n(x) \times n(z)}^{\prime}, \Omega^{\prime}\right]^{\prime}$ and denote the solution of the model more compactly by

$$
\begin{aligned}
\mathbf{w}_{t+1} & =\mathbf{h}^{w}\left(\mathbf{w}_{t}, \sigma\right)+\sigma \tilde{\Omega} \boldsymbol{\epsilon}_{t+1}=\left[\begin{array}{c}
\mathbf{h}^{x}\left(\mathbf{w}_{\mathbf{t}}, \sigma\right) \\
\mathbf{h}^{z}\left(\mathbf{w}_{\mathbf{t}}, \sigma\right)+\sigma \Omega \boldsymbol{\epsilon}_{t+1}
\end{array}\right], \\
\mathbf{y}_{t} & =\mathbf{h}^{y}\left(\mathbf{w}_{t}, \sigma\right) .
\end{aligned}
$$

Then by defining

$$
\tilde{\mathbf{g}}\left(\mathbf{w}_{t}, \mathbf{y}_{t}, \mathbf{w}_{t+1}, \mathbf{y}_{t+1}\right)=\left[\begin{array}{c}
\mathbf{g}\left(\mathbf{x}_{t}, \mathbf{z}_{t}, \mathbf{y}_{t}, \mathbf{x}_{t+1}, \mathbf{z}_{t+1}, \mathbf{y}_{t+1}\right) \\
\mathbf{z}_{t+1}-\Pi \mathbf{z}_{t}
\end{array}\right],
$$

(1) can be written as

$$
\begin{aligned}
& \mathbf{0}_{n(w)+n(y)}=\mathbf{E}_{t} \tilde{\mathbf{g}}\left(\mathbf{w}_{t}, \mathbf{h}^{y}\left(\mathbf{w}_{t}, \sigma\right), \mathbf{h}^{w}\left(\mathbf{w}_{t}, \sigma\right)+\sigma \tilde{\Omega} \boldsymbol{\epsilon}_{t+1}, \mathbf{h}^{y}\left(\mathbf{h}^{w}\left(\mathbf{w}_{t}, \sigma\right)\right.\right. \\
& \left.\left.\quad+\sigma \tilde{\Omega} \boldsymbol{\epsilon}_{t+1}, \sigma\right)\right) \\
& =\mathbf{G}\left(\mathbf{w}_{t}, \sigma\right) .
\end{aligned}
$$

According to the implicit function theorem, the partial derivatives of $\mathbf{G}$ with respect to $\mathbf{w}_{t}$ must vanish at the stationary solution $\mathbf{w}$ obtained from $\sigma=0$. This yields a system of equations in the $(n(w)+n(y)) \times n(w)$ coefficients of the linear part of $\mathbf{h}^{w}$ and $\mathbf{h}^{y}$, denoted by $L^{w}$ and $L^{y}$, respectively:

$$
\begin{aligned}
0 & =\tilde{g}_{w_{j t}}^{i}+\sum_{l=1}^{n(y)} \tilde{g}_{y_{l t}}^{i} L_{l, j}^{y}+\sum_{l=1}^{n(w)} \tilde{g}_{w_{l t+1}}^{i} L_{l, j}^{w}+\sum_{l=1}^{n(y)} \tilde{g}_{y_{l+1}}^{i} \sum_{k=1}^{n(w)} L_{l, k}^{y} L_{k, j}^{w}, \\
i & =1, \ldots, n(w)+n(y), \\
j & =1, \ldots, n(w) .
\end{aligned}
$$

One must pick the solution of this system for which the eigenvalues of the matrix $L^{w}$ are within the unit circle, so that the linearized dynamic system $\mathbf{w}_{t+1}=$ $\mathbf{w}+L^{w}\left(\mathbf{w}_{t}-\mathbf{w}\right)$ is stable.

\subsection{The $A B$ Model and the $Q Z$ Factorization}

A second way to obtain the matrices $L^{w}$ and $L^{y}$ is to linearize the system (1) at the point $(\mathbf{x}, \mathbf{0}, \mathbf{y})$, solving $\mathbf{g}(\mathbf{x}, \mathbf{0}, \mathbf{y}, \mathbf{x}, \mathbf{y}, \mathbf{0})=\mathbf{0}_{n(x)+n(y)}$. This yields the system 
of linear stochastic difference equations

$$
\begin{aligned}
& A \mathbf{E}_{t}\left[\begin{array}{c}
\overline{\mathbf{w}}_{t+1} \\
\overline{\mathbf{y}}_{t+1}
\end{array}\right]=B\left[\begin{array}{c}
\overline{\mathbf{w}}_{t} \\
\overline{\mathbf{y}}_{t}
\end{array}\right], \quad \overline{\mathbf{w}}_{t} \equiv\left[\begin{array}{c}
\mathbf{x}_{t}-\mathbf{x} \\
\mathbf{z}_{t}
\end{array}\right], \quad \overline{\mathbf{y}}_{t} \equiv \mathbf{y}_{t}-\mathbf{y}, \\
& A=\left[\begin{array}{ccc}
\mathbf{g}_{4} & \mathbf{g}_{5} & \mathbf{g}_{6} \\
\mathbf{0}_{n(z) \times n(x)} & I_{n(z)} & \mathbf{0}_{n(z) \times n(y)}
\end{array}\right], \\
& B=\left[\begin{array}{ccc}
-\mathbf{g}_{1} & -\mathbf{g}_{2} & -\mathbf{g}_{3} \\
\mathbf{0}_{n(z) \times n(y)} & \Pi & \mathbf{0}_{n(z) \times n(y)}
\end{array}\right],
\end{aligned}
$$

where $\mathbf{g}_{i}$ denotes the Jacobian matrix of $\mathbf{g}$ with respect to its $i$ th argument.

Usually, the linear system (4) contains a number of equations that involve only variables dated at time $t$. These arise from equations such as the economy's resource constraint or from static first-order conditions. In this case the matrix $A$ is singular, so that $A^{-1} B$ does not exist and the procedure outlined by Blanchard and Kahn (1980) cannot be applied. ${ }^{4}$ As pointed out by Klein (2000), QZ factorization can be used to solve the system (4). ${ }^{5}$

There are two ways to use QZ factorization to solve the model (4a). As shown in Heiberger et al. (2015), both provide the same solution (if it exists at all). The first way [see Klein (2000)] rests on factoring the matrix pencil $(B-\lambda A)$, the second on factoring $(A-\mu B)$ [see Heer and Maußner (2009)]. The QZ factorization of the pencil $(B-\lambda A)$ is

$$
\begin{aligned}
& Q^{\mathrm{H}} A Z=S, \\
& Q^{\mathrm{H}} B Z=T,
\end{aligned}
$$

where $Q$ and $Z$ are unitary matrices, $S$ and $T$ are upper triangular matrixes, and $Q^{\mathrm{H}}$ denotes the Hermitian transpose of $Q .{ }^{6}$ The eigenvalues of the matrix pencil are given by $\lambda_{i}=t_{i i} / s_{i i}$ for $s_{i i} \neq 0 .^{7}$ Furthermore, the matrices $S$ and $T$ can be arranged so that the eigenvalues appear in ascending order with respect to their absolute values. Assume that $n(w)=n(x)+n(z)$ eigenvalues have modulus less than one and $n(y)$ have modulus greater than one. Let $Z_{11}$ denote the upper left $n(w) \times n(w)$ block of $Z, Z_{12}$ the upper right $n(w) \times n(y)$ block, etc., and define new variables

$$
\left[\begin{array}{ll}
Z_{11} & Z_{12} \\
Z_{21} & Z_{22}
\end{array}\right]\left[\begin{array}{c}
\tilde{\mathbf{w}}_{t} \\
\tilde{\mathbf{y}}_{t}
\end{array}\right]=\left[\begin{array}{c}
\overline{\mathbf{w}}_{t} \\
\overline{\mathbf{y}}_{t}
\end{array}\right]
$$

so that we can write (4) as

$$
\left[\begin{array}{cc}
S_{11} & S_{12} \\
0_{n(y) \times n(w)} & S_{22}
\end{array}\right] \mathbf{E}_{t}\left[\begin{array}{c}
\tilde{\mathbf{w}}_{t+1} \\
\tilde{\mathbf{y}}_{t+1}
\end{array}\right]=\left[\begin{array}{cc}
T_{11} & T_{12} \\
0_{n(y) \times n(w)} & T_{22}
\end{array}\right]\left[\begin{array}{c}
\tilde{\mathbf{w}}_{t} \\
\tilde{\mathbf{y}}_{t}
\end{array}\right] .
$$

$S_{11}$ is an $n(w) \times n(w)$ upper triangular matrix, $S_{22}$ is an $n(y) \times n(y)$ upper triangular matrix, and $S_{12}$ is an $n(w) \times n(y)$ matrix. The matrices $T_{11}, T_{22}$, and $T_{12}$ have corresponding dimensions. 
Given these assumptions and definitions, the system

$$
S_{22} \mathbf{E}_{t} \tilde{\mathbf{y}}_{t+1}=T_{22} \tilde{\mathbf{y}}_{t}
$$

is unstable ${ }^{8}$ and to obtain a definite solution, we must set $\tilde{\mathbf{y}}_{t}=\mathbf{0}_{n(y)}$ for all $t$. Thus, from the first line of (7),

$$
\tilde{\mathbf{w}}_{t+1}=S_{11}^{-1} T_{11} \tilde{\mathbf{w}}_{t} .
$$

Because

$$
\tilde{\mathbf{w}}_{t}=Z_{11}^{-1} \overline{\mathbf{w}}_{t}
$$

from the first line of (6), we get

$$
\overline{\mathbf{w}}_{t+1}=\underbrace{Z_{11} S_{11}^{-1} T_{11} Z_{11}^{-1}}_{L^{w}} \overline{\mathbf{w}}_{t} .
$$

The second line of (6), together with (8), implies that

$$
\overline{\mathbf{y}}_{t}=\underbrace{Z_{21} Z_{11}^{-1}}_{L^{y}} \overline{\mathbf{w}}_{t} .
$$

The dynamics of the solved linear model can be represented by

$$
\begin{aligned}
& \overline{\mathbf{x}}_{t+1}=L_{x}^{x} \overline{\mathbf{x}}_{t}+L_{z}^{x} \mathbf{z}_{t}, \\
& \overline{\mathbf{y}}_{t+1}=L_{x}^{y} \overline{\mathbf{x}}_{t}+L_{z}^{y} \mathbf{z}_{t}, \\
& \mathbf{z}_{t+1}=\Pi \mathbf{z}_{t}+\sigma \Omega \boldsymbol{\epsilon}_{t+1},
\end{aligned}
$$

where the matrices of the linear approximation of the policy functions (2) are related to $L^{w}$ and $L^{y}$ according to

$$
L^{w}=\left[\begin{array}{cc}
L_{x}^{x} & L_{z}^{x} \\
\mathbf{0}_{n(z) \times n(x)} & \Pi
\end{array}\right], \quad L^{y}=\left[\begin{array}{ll}
L_{x}^{y} & L_{z}^{y}
\end{array}\right] .
$$

\subsection{Model Reduction}

In this subsection we assume that the researcher is able to sort the equations in $\mathbf{g}(\cdot)$ so that the first $n(u)$ equations involve only period- $t$ variables. This allows us to partition $\mathbf{y}_{t}=\left[\mathbf{u}_{t}^{\prime}, \mathbf{v}_{t}^{\prime}\right]^{\prime}$ and to write the linearized system (1) as

$$
\begin{gathered}
C_{u} \overline{\mathbf{u}}_{t}=C_{w v}\left[\begin{array}{c}
\overline{\mathbf{w}}_{t} \\
\overline{\mathbf{v}}_{t}
\end{array}\right], \\
D_{w v} \mathbf{E}_{t}\left[\begin{array}{c}
\overline{\mathbf{w}}_{t+1} \\
\overline{\mathbf{v}}_{t+1}
\end{array}\right]+F_{w v}\left[\begin{array}{c}
\overline{\mathbf{w}}_{t} \\
\overline{\mathbf{v}}_{t}
\end{array}\right]=\tilde{D}_{u} \mathbf{E}_{t} \overline{\mathbf{u}}_{t+1}+\tilde{F}_{u} \overline{\mathbf{u}}_{t},
\end{gathered}
$$


where the matrices are related to the Jacobian matrix of $\mathbf{g}$ according to

$$
\begin{aligned}
D \mathbf{g} & =\left[\begin{array}{llllllll}
C_{x} & C_{z} & C_{u} & C_{v} & 0 & 0 & 0 & 0 \\
F_{x} & F_{z} & F_{u} & F_{v} & D_{x} & D_{z} & D_{u} & D_{v}
\end{array}\right], \\
C_{w v} & =\left[\begin{array}{lll}
-C_{x} & -C_{z}-C_{v}
\end{array}\right], D_{w v}=\left[\begin{array}{ccc}
D_{x} & D_{z} & D_{v} \\
0 & I_{n(z)} & 0
\end{array}\right], F_{w v}=\left[\begin{array}{ccc}
F_{x} & F_{z} & F_{v} \\
0 & -\Pi & 0
\end{array}\right], \\
\tilde{D}_{u} & =\left[\begin{array}{c}
D_{u} \\
0
\end{array}\right], \tilde{F}_{u}=\left[\begin{array}{c}
F_{u} \\
0
\end{array}\right] .
\end{aligned}
$$

Solving (10a) for $\mathbf{u}_{t}$ and using the result in (10b) yields

$$
\mathbf{E}_{t}\left[\begin{array}{c}
\overline{\mathbf{w}}_{t+1} \\
\overline{\mathbf{v}}_{t+1}
\end{array}\right]=W\left[\begin{array}{c}
\overline{\mathbf{w}}_{t} \\
\overline{\mathbf{v}}_{t}
\end{array}\right], W=\left[D_{w v}-\tilde{D}_{u} C_{u}^{-1} C_{w v}\right]^{-1}\left[F_{w v}-\tilde{F}_{u} C_{u}^{-1} C_{w v}\right] .
$$

This system can be solved along the same lines as the system (4a). We present the necessary steps in the Online Appendix.

The solutions of (4) and (10) also solve (3). To see this, let $\overline{\mathbf{w}}_{t+1}=L^{w} \overline{\mathbf{w}}_{t}$ and $\overline{\mathbf{y}}_{t}=L^{y} \overline{\mathbf{w}}_{t}$ denote the solution of (4). This yields

$$
A\left[\begin{array}{c}
L^{w} \\
L^{y} L^{w}
\end{array}\right] \overline{\mathbf{w}}_{t}-B\left[\begin{array}{c}
I_{n(w)} \\
L^{y}
\end{array}\right] \overline{\mathbf{w}}_{t}=\mathbf{0}_{n(w)+n(y)} .
$$

The coefficients of $L^{w}$ and $L^{y}$ thus must satisfy the system of equations

$$
A\left[\begin{array}{c}
L^{w} \\
L^{y} L^{w}
\end{array}\right]-B\left[\begin{array}{c}
I_{n(w)} \\
L^{y}
\end{array}\right]=\mathbf{0}_{(n(w)+n(y)) \times n(w)},
$$

which is just the matrix version of (3). Note that (12) provides a simple way to check the accuracy of solutions based on matrix methods against the solution that would solve the nonlinear system (3). Given the matrices $A$ and $B$ and the policy functions $L^{w}$ and $L^{y}$, the entries of the $(n(w)+n(y)) \times n(w)$ matrix on the left-hand side of (12) should not exceed a given tolerance, $10^{-6}$, say, in absolute value.

\subsection{Implementation}

The linear algebra package LAPACK provides several routines to compute both the QZ factorization of a matrix pencil and the Schur decomposition of nonsymmetric matrices. ${ }^{9}$ The Fortran program Solab by Paul Klein, which is also used by DYNARE, employs the LAPACK routine ZGGES to factor the pencil $(B-\lambda A)$. We use the LAPACK routines ZGGESX and ZGGESXV. Both ZGGES and ZGGESX compute the matrices $S, T, Q$, and $Z$ and cluster the eigenvalues in two blocks. The eigenvalues are given by $\lambda_{i}=\alpha_{i} / \beta_{i}$, where $\alpha_{i}$ and $\beta_{i}$ are equal to the diagonal elements of $S$ and $T$, respectively. Klein's procedure requires clustering the eigenvalues according to the criterion $\left|\alpha_{i}\right|>\left|\beta_{i}\right|$ (or $\left|\lambda_{i}\right|>1$ ) so 
that the eigenvalues of $S_{11}^{-1} T_{11}$ are within the unit circle. ${ }^{10}$ ZGGESX additionally computes average reciprocal condition numbers $\kappa$ for the eigenvalue clusters. An approximate error bound for each cluster is given by ${ }^{11}$

$$
\chi\left(\bar{\lambda}, \bar{\lambda}^{\prime}\right)=\frac{\left|\bar{\alpha} \bar{\beta}^{\prime}-\bar{\beta} \bar{\alpha}^{\prime}\right|}{\sqrt{|\bar{\alpha}|^{2}+|\bar{\beta}|^{2}} \sqrt{\left|\bar{\alpha}^{\prime}\right|^{2}+\left|\bar{\beta}^{\prime}\right|^{2}}} \leq \frac{\epsilon \sqrt{\|A\|_{1}^{2}+\|B\|_{1}^{2}}}{\kappa},
$$

where $\chi$ is the chordal distance between the average eigenvalue in the selected cluster $\bar{\lambda}=\sum_{i=1}^{n(w)} \lambda_{i} /(n(w))$ and the average true eigenvalue $\bar{\lambda}^{\prime}$ of the pencil. $\epsilon$ refers to the machine epsilon, $\|A\|_{1}$ and $\|B\|_{1}$ are the one-norms of $A$ and $B$, respectively. They are returned from ZGGESXV. The routines ZGGES and ZGGESX balance $A$ and $B$ by scaling the rows and columns to reduce computational errors. Therefore, the error bounds refer to the scaled matrices and not to $A$ and $B$ as passed to ZGGES and ZGGESX. As will become apparent later, an additional scaling may be necessary to reduce computational errors.

The LAPACK routine ZGEESX performs the Schur decomposition $W=Z S Z^{\mathrm{H}}$ of the matrix $W$ in (11), clusters eigenvalues so that the eigenvalues of $S_{11}$ are within the unit circle, and provides reciprocal condition numbers $\kappa_{i}, i=1,2$, for the average eigenvalues of $S_{11}$ and $S_{22}$. An approximate error bound for the distance between the true average eigenvalue $\bar{\lambda}^{\prime}$ of $S_{11}$ and the computed average $\bar{\lambda}$ is given by

$$
\left|\bar{\lambda}-\bar{\lambda}^{\prime}\right| \leq \frac{\epsilon|| W \|_{1}}{\kappa_{1}}
$$

As in the case of the generalized eigenvalue problem, ZGEESX scales the rows and columns of the matrix $W$ to reduce the computational errors. Therefore, the one-norm of $W$ returned by ZGEEVX is the norm of the scaled matrix.

\section{AN EXAMPLE}

We consider a real business cycle model taken from Heer and Maußner (2013) that features habits in consumption and hours as well as adjustment costs of capital. The model introduces endogenous labor supply into the model of Jermann (1998), who studied the equity premium implications of a production economy.

\subsection{The Model}

Households. Households enter the current period $t$ with given amounts of firm shares $S_{t}$ and real bonds $B_{t}$. Bonds have a maturity of one period and can be purchased at the current price $v_{t}^{b}$ and pay one unit of consumption in period $t+1$. The real share price is $v_{t}^{e}$ and real dividend payments per share are $d_{t}$. Firms pay the real wage $w_{t}$ per unit of working hours $N_{t}$. Thus,

$$
v_{t}^{e}\left(S_{t+1}-S_{t}\right)+v_{t}^{b} B_{t+1} \leq w_{t} N_{t}+d_{t} S_{t}+B_{t}-C_{t}
$$


is the household's budget constraint, where $C_{t}$ denotes consumption. The household chooses contingency plans for consumption $C_{t}$, hours $N_{t}$, and next-period stocks $S_{t+1}$ that maximize

$$
U_{t}=\mathbf{E}_{t} \sum_{s=0}^{\infty} \beta^{s} \frac{\left(C_{t+s}-\chi^{C} C_{t+s-1}\right)^{1-\eta}-1}{1-\eta}-v_{0} \frac{\left(N_{t+s}-\chi^{N} N_{t+s-1}\right)^{1+\nu_{1}}-1}{1+v_{1}}
$$

subject to (15). The parameters $\chi^{C}$ and $\chi^{N}$ determine the degree of habits in consumption and labor supply. We treat both habits as exogenous; i.e., $C_{t+s-1}$ and $N_{t+s-1}$ refer to the average consumption and labor supply of the previous period. The first-order conditions for this problem and any further mathematical details of this model are presented in the Online Appendix.

Firms. The representative firm uses labor $N_{t}$ and capital $K_{t}$ to produce output $Y_{t}$ according to the production function

$$
Y_{t}=Z_{t} N_{t}^{1-\alpha} K_{t}^{\alpha}, \quad \alpha \in(0,1) .
$$

The level of total factor productivity $Z_{t}$ is governed by the AR(1) process

$$
\ln Z_{t}=\rho \ln Z_{t-1}+\sigma \epsilon_{t}, \quad \epsilon_{t} \sim \mathcal{N}(0,1) .
$$

The firm finances part of its investment $I_{t}$ from retained earnings $\mathrm{RE}_{t}$ and issues new shares to cover the remaining part:

$$
I_{t}=v_{t}\left(S_{t+1}-S_{t}\right)+\mathrm{RE}_{t} .
$$

It distributes the excess of its profits over retained earnings to the household sector:

$$
d_{t} S_{t}=Y_{t}-w_{t} N_{t}-\mathrm{RE}_{t} .
$$

Investment increases the firm's future stock of capital according to

$$
K_{t+1}=\Phi\left(I_{t} / K_{t}\right) K_{t}+(1-\delta) K_{t}, \quad \delta \in[0,1],
$$

where we parameterize the function $\Phi$ as

$$
\Phi\left(I_{t} / K_{t}\right):=\frac{a_{1}}{1-\zeta}\left(\frac{I_{t}}{K_{t}}\right)^{1-\zeta}+a_{2}, \quad \zeta>0,
$$

and determine $a_{1}$ and $a_{2}$ from $\Phi^{\prime}(\delta)=1$ and $\Phi(\delta)=\delta$ so that adjustment costs are absent in the deterministic stationary equilibrium.

The firm maximizes its beginning-of-period value

$$
V_{t}=\mathbf{E}_{t} \sum_{s=0}^{\infty} \varrho_{t+s}\left(Y_{t+s}-w_{t+s} N_{t+s}-I_{t+s}\right)
$$


TABLE 1. Parameter choice

\begin{tabular}{|c|c|c|c|c|}
\hline Preferences & $\begin{aligned} \beta & =0.99 \\
\chi^{C} & =0.82\end{aligned}$ & $\begin{aligned} \eta & =5 \\
\chi^{N} & =0.82\end{aligned}$ & $v_{1}=2.5$ & $N \in\{0.13,1 / 3\}$ \\
\hline Production & $\alpha=0.36$ & $\rho=0.95$ & $\sigma=0.00712$ & \\
\hline Capital accumulation & $\delta=0.025$ & $\zeta=1 / 0.23$ & & \\
\hline
\end{tabular}

subject to (17) and (21). The variable

$$
\varrho_{t+s}=\beta^{s} \frac{\Lambda_{t+s}}{\Lambda_{t}}
$$

is the household's stochastic discount factor for period- $(t+s)$ returns, and $\Lambda_{t}$ equals the marginal utility of consumption:

$$
\Lambda_{t}=\left(C_{t}-\chi^{C} C_{t-1}\right)^{-\eta}
$$

The respective first-order conditions can be found in the Online Appendix.

Calibration. We calibrate the model with respect to the U.S. economy. Table 1 displays our choice of parameters. The standard parameter values for the production side, $\alpha, \rho$, and $\sigma$, are taken from Hansen (1985), as well as the value of the discount factor $\beta$. The habit parameter $\chi^{C}, \eta$, and the parameters of the capital accumulation equation (21) are taken from Jermann (1998). The parameter $v_{1}=2.5$ is from De Paoli et al. (2010). Like these authors, we assume that $\chi^{N}=\chi^{C 12}$ and choose $v_{0}$ so that at the stationary equilibrium $N$ equals $1 / 3$.

\subsection{Accuracy of the Solution}

Solutions. In this section we consider the linear part of the model's approximate solution. We compute four different linear solutions for the levels of the variables:

1. the solution of the nonlinear system via a nonlinear equations solver, with termination criterion $\max _{k}\left|f_{k}\left(L^{w}, L^{y}\right)\right|<10^{-7}$, where $f_{k}, k=1, \ldots,(n(w)+n(y)) \times$ $n(w)$ denotes the $k$ th equation of (3),

2. the solution via the $\mathrm{QZ}$ factorization of the matrix pencil $(B-\lambda A)$,

3. the solution via the $\mathrm{QZ}$ factorization of the matrix pencil $(A-\mu B)$,

4. the solution of the reduced system, where we partition

$$
\mathbf{y}_{t}=\left[\mathbf{u}_{t}^{\prime}, \mathbf{v}_{t}^{\prime}\right]^{\prime}=\left[Y_{t}, C_{t}, I_{t}, N_{t}, w_{t}, q_{t}, \Lambda_{t}\right]^{\prime}
$$

so that $\mathbf{v}_{\mathbf{t}} \equiv \Lambda_{t} \cdot{ }^{13}$

As shown in Section 2, if we ignore different degrees of numerical precision, all four solutions should deliver the same policy functions.

Here we report the solutions from our Fortran program. On our home page we also provide a Maple program and a DYNARE script. ${ }^{14}$ Whereas the Fortran 
program employs a central difference approximation of the Jacobian matrix of $\mathbf{g}$, the Maple program and DYNARE use symbolic algebra to derive the analytic formulas for the elements of the Jacobian and evaluate these at the stationary solution. The results reported in the following are robust with respect to the computation of the Jacobian. The policy functions from DYNARE reproduce solution \#2.

With these solutions we also simulate the model and report second moments for the percentage deviations of the model's variables from their respective steady state values. ${ }^{15}$ We do the latter for three reasons. First, researchers usually do not report the coefficients of the policy functions but present statistics that summarize the empirical implications of their models. For this reason, it is important to see whether or not simulated measures of the business cycle bear traces of the numerical differences in the coefficients of the policy functions. Second, as is well known from the benchmark business cycle model, different degrees of numerical accuracy rarely surface in differences of second moments from simulated time series. ${ }^{16}$ Third, as we demonstrate in the Online Appendix, if we solved and simulated the log-linearized version of the model, the respective second moments would not depend on the value of $N$, the stationary fraction of hours supplied by the representative agent. Therefore, any discrepancies we observe indicate serious numerical differences between the four different solutions.

Policy functions. Tables A.2 and A.3 in the Online Appendix report the coefficients of the policy functions (i.e., the linear approximate solution of the model) for the cases $N=0.13$ and $N=1 / 3$. To save on space, here we focus on the relative difference between solutions \#2 through \#4 and solution \#1.

In the case $N=1 / 3$ the coefficients are virtually identical: the maximum relative difference between the coefficients is less than $0.005 \%$ and relates to the coefficient of Tobin's $q$ with respect to the capital stock, as computed by solution \# 3. This changes considerably if we use $N=0.13$, a value used by Heer and Maußner (2013) for the German economy.

Table 2 presents the results for this case. There are virtually no differences between the solution of the reduced system and the nonlinear solution. In absolute terms the maximum relative distance between the solution based on the QZ factorization $(B-\lambda A)$ and the nonlinear solutions is $65 \%$ for the coefficient of $q_{t}$ with respect to capital $K_{t}$ : the nonlinear solution provides a coefficient of about -0.024 , whereas solution \#2 yields about -0.008 . For the same coefficient, the QZ factorization of $(B-\mu A)$ (solution \#3) even delivers a positive value of about 0.014 , which gives raise to a relative difference of over $150 \%$. Whereas the nonlinear solution gives positive coefficients in the policy function for the future capital stock $K_{t+1}$ for $N_{t-1}$ and $\ln Z_{t}$ of 0.85 and 0.22 , respectively, solution \#3 yields zero coefficients, which explains the $100 \%$ deviation in the entries for $K_{t+1}$ in Table 2.

Second moments. Table 3 presents results from six different simulations of the model. The moments in the first and second panels rest on solutions \#2 and \#3, 
TABLE 2. Policy functions for $N=0.13$, relative discrepancy

\begin{tabular}{|c|c|c|c|c|}
\hline \multirow{2}{*}{$\begin{array}{l}\text { Dependent } \\
\text { variables }\end{array}$} & \multicolumn{4}{|c|}{ Independent variables } \\
\hline & $K_{t}$ & $C_{t-1}$ & $N_{t-1}$ & $\ln Z_{t}$ \\
\hline & \multicolumn{4}{|c|}{$(B-\lambda A)$ versus nonlinear system } \\
\hline$K_{t+1}$ & 0.000446 & 0.005025 & 0.004494 & 0.017461 \\
\hline$Y_{t}$ & 0.009649 & -0.003991 & 0.001928 & 0.007353 \\
\hline$C_{t}$ & -0.036036 & 0.001049 & -0.003646 & -0.013363 \\
\hline$I_{t}$ & 0.018323 & 0.005025 & 0.004494 & 0.017461 \\
\hline$N_{t}$ & -0.045245 & -0.003991 & 0.001928 & -0.015204 \\
\hline$w_{t}$ & -0.004071 & -0.003991 & 0.001928 & -0.002356 \\
\hline$q_{t}$ & -0.650130 & 0.005025 & 0.004494 & 0.017461 \\
\hline \multirow[t]{2}{*}{$\Lambda_{t}$} & -0.036036 & -0.003991 & -0.003646 & -0.013363 \\
\hline & \multicolumn{4}{|c|}{$(A-\mu B)$ versus nonlinear system } \\
\hline$K_{t+1}$ & -0.008139 & -1.019427 & -1.000000 & -1.000000 \\
\hline$Y_{t}$ & 0.027064 & -0.018830 & 0.009491 & 0.023591 \\
\hline$C_{t}$ & -0.101083 & 0.004949 & -0.017945 & -0.042873 \\
\hline$I_{t}$ & 0.043836 & 0.003276 & 0.002099 & 0.035338 \\
\hline$N_{t}$ & -0.126914 & -0.018830 & 0.009491 & -0.048780 \\
\hline$w_{t}$ & -0.011419 & -0.018830 & 0.009491 & -0.007558 \\
\hline$q_{t}$ & -1.566015 & 0.004084 & 0.002891 & 0.036156 \\
\hline$\Lambda_{t}$ & -0.101083 & -0.018830 & -0.017945 & -0.042873 \\
\hline
\end{tabular}

Reduced system versus nonlinear system

\begin{tabular}{lllll}
\cline { 2 - 5 }$K_{t+1}$ & 0.000000 & 0.000000 & 0.000000 & 0.000000 \\
$Y_{t}$ & 0.000000 & 0.000000 & 0.000000 & 0.000000 \\
$C_{t}$ & 0.000000 & 0.000000 & 0.000000 & 0.000000 \\
$I_{t}$ & 0.000000 & 0.000000 & 0.000000 & 0.000000 \\
$N_{t}$ & 0.000000 & 0.000000 & 0.000000 & 0.000000 \\
$w_{t}$ & 0.000000 & 0.000000 & 0.000000 & 0.000000 \\
$q_{t}$ & 0.000001 & 0.000000 & 0.000000 & 0.000000 \\
$\Lambda_{t}$ & 0.000000 & 0.000000 & 0.000000 & 0.000000 \\
\hline
\end{tabular}

Notes: The entries represent relative differences between the coefficients of the policy functions of the variables in the leftmost column. $\Lambda_{t}$ is the Lagrange multiplier of the household's budget constraint.

respectively, whereas the moments in the third panel are from simulations that use the policy function obtained from the nonlinear solution \#1. The single difference between the panels labeled $N=1 / 3$ and $N=0.13$ is two different values for the stationary level of hours $N$. The second moments refer to percentage deviations of a variable from its stationary solution. They were computed as averages over 500 simulations. Each individual time series has 200 observations. We show in the Online Appendix that the coefficient matrices of the log-linearized system do not depend on $N$, so that the simulations should yield identical second moments, given that the same sequence of random numbers is used. Obviously, this is true 
TABLE 3. Second moments

\begin{tabular}{|c|c|c|c|c|c|c|c|c|}
\hline \multirow[b]{2}{*}{ Variable } & \multicolumn{4}{|c|}{$N=1 / 3$} & \multicolumn{4}{|c|}{$N=0.13$} \\
\hline & $s_{x}$ & $s_{x} / s_{Y}$ & $r_{x Y}$ & $r_{x}$ & $s_{x}$ & $s_{x} / s_{Y}$ & $r_{x Y}$ & $r_{x}$ \\
\hline & \multicolumn{8}{|c|}{$(A-\mu B)$} \\
\hline Output & 1.19 & 1.00 & 1.00 & 0.90 & 0.82 & 1.00 & 1.00 & 0.79 \\
\hline Consumption & 1.09 & 0.91 & 0.97 & 0.97 & 0.34 & 0.41 & 1.00 & 0.79 \\
\hline Investment & 1.77 & 1.48 & 0.90 & 0.67 & 2.17 & 2.65 & 1.00 & 0.79 \\
\hline Hours & 1.37 & 1.15 & -0.97 & 0.96 & 1.92 & 2.34 & -0.84 & 0.98 \\
\hline Real wage & 2.55 & 2.13 & 0.99 & 0.94 & 2.65 & 3.23 & 0.92 & 0.94 \\
\hline \multirow[t]{2}{*}{ Tobin's $q$} & 7.09 & 5.93 & 0.79 & 0.61 & 9.46 & 11.54 & 1.00 & 0.79 \\
\hline & \multicolumn{8}{|c|}{$(B-\lambda A)$} \\
\hline Output & 1.19 & 1.00 & 1.00 & 0.90 & 0.82 & 1.00 & 1.00 & 0.75 \\
\hline Consumption & 1.09 & 0.91 & 0.97 & 0.97 & 0.70 & 0.86 & 0.94 & 0.95 \\
\hline Investment & 1.77 & 1.48 & 0.90 & 0.67 & 1.44 & 1.75 & 0.89 & 0.31 \\
\hline Hours & 1.37 & 1.15 & -0.97 & 0.96 & 1.98 & 2.42 & -0.88 & 0.96 \\
\hline Real wage & 2.55 & 2.13 & 0.99 & 0.94 & 2.73 & 3.34 & 0.94 & 0.94 \\
\hline \multirow[t]{2}{*}{ Tobin's $q$} & 7.09 & 5.93 & 0.79 & 0.61 & 5.97 & 7.29 & 0.80 & 0.24 \\
\hline & \multicolumn{8}{|c|}{ Nonlinear solution } \\
\hline Output & 1.19 & 1.00 & 1.00 & 0.90 & 1.19 & 1.00 & 1.00 & 0.90 \\
\hline Consumption & 1.09 & 0.91 & 0.97 & 0.97 & 1.09 & 0.91 & 0.97 & 0.97 \\
\hline Investment & 1.77 & 1.48 & 0.90 & 0.67 & 1.77 & 1.48 & 0.90 & 0.67 \\
\hline Hours & 1.37 & 1.15 & -0.97 & 0.96 & 1.37 & 1.15 & -0.97 & 0.96 \\
\hline Real wage & 2.55 & 2.13 & 0.99 & 0.94 & 2.55 & 2.13 & 0.99 & 0.94 \\
\hline Tobin's $q$ & 7.09 & 5.93 & 0.79 & 0.61 & 7.09 & 5.93 & 0.79 & 0.61 \\
\hline
\end{tabular}

Notes: $s_{x}=$ Standard deviation of percentage deviations of variable $x$ from its stationary solution. $x$ stands for any of the variables from column (1). Results are from 500 replications with 200 observations each. $s_{x} / s_{Y}=$ Standard deviation of variable $x$ relative to standard deviation of output $Y . r_{x Y}=$ Cross correlation of variable $x$ with output $y . r_{x}=$ First-order autocorrelation of variable $x$.

for the nonlinear solution (and also, but not shown in the Table, for solution \#4). However, the second moments obtained from solutions \#2 and \#3 reveal many obvious and large differences, both in the standard deviations and in the cross and autocorrelations of the variables displayed.

Euler equation residuals. Euler equation residuals are frequently employed to investigate the degree of numerical accuracy. In Table 4 we report the maximum residuals of the Euler equation for capital: ${ }^{17}$

$$
\Lambda_{t}=\beta \mathbf{E}_{t} \frac{\Lambda_{t+1}\left[\alpha\left(Y_{t+1} / K_{t+1}\right)-\left(I_{t+1} / K_{t+1}\right)+q_{t+1}\left(1-\delta+\Phi\left(I_{t+1} / K_{t+1}\right)\right)\right]}{q_{t}} .
$$


TABLE 4. Euler equation residuals

\begin{tabular}{lcc}
\hline Solution & $N=1 / 3$ & $N=0.13$ \\
\hline$\# 1$ & 0.04519 & 0.04519 \\
$\# 2$ & 0.04519 & 0.04711 \\
$\# 3$ & 0.04519 & 0.04860 \\
$\# 4$ & 0.04519 & 0.04519 \\
\hline
\end{tabular}

Notes: The leftmost column indicates the number of the solution as defined in the body of the paper.

We compute the residuals on a grid $\mathscr{G}$ defined on the four-dimensional cube around the stationary solution of the variables $K, C, N$, and $Z=1$ given by ${ }^{18}$

$$
[0.93 K, 1.07 K] \times[0.93 C, 1.07 C] \times[0.94 N, 1.04 N] \times[0.95,1.05] .
$$

Each of the subintervals is discretized in 50 points. For each $\left(K_{t}, C_{t-1}, N_{t-1}, Z_{t}\right) \in$ $\mathscr{G}$, we evaluate the right-hand side of equation (25) by Gauss-Hermite integration with four points and use the respective value of $\Lambda_{t}$ to compute the amount of consumption $\hat{C}_{t}$ that would deliver a zero residual from equation (24). The residual is defined as $\left(\hat{C}_{t} / C_{t}\right)-1$, where $C_{t}$ is the amount of consumption determined from the policy function [see Judd and Guu (1997)].

The Euler equation residuals confirm the results from the simulations, albeit they are not as impressive. ${ }^{19}$ Solution \#3 obtained from the QZ decomposition of $(A-\mu B)$ is the least accurate, followed by solution \#2. The consumption equivalent of the former (latter) is about $4.9 \%$ (4.7), as compared to $4.5 \%$ for the more accurate nonlinear solution \#1 and the solution of the reduced system. For the case $N=1 / 3$, all four solutions deliver the same Euler residual.

\subsection{Source of the Problem and Remedies}

Unbalanced matrices. The odd results reported in the previous subsection originate in two equations, the first-order conditions for consumption and for labor supply:

$$
\begin{aligned}
\Lambda_{t} & =\left(C_{t}-\chi^{C} C_{t-1}\right)^{-\eta}, \\
\Lambda_{t} w_{t} & =v_{0}\left(N_{t}-\chi^{N} N_{t-1}\right)^{\nu_{1}} .
\end{aligned}
$$

The steady-state value of consumption is low and increases with the stationary value of working hours, $N$. Therefore, a strong habit ( $\chi^{C}$ close to one) and a large coefficient of relative risk aversion $\eta$ imply a huge value of $\Lambda$, the multiplier of the budget constraint (15). This gives rise to very large coefficients in the Jacobian matrix of $\mathbf{g}$, and, accordingly, in the matrix $B$ of (4a) and the matrix $W$ of (11). Yet, because of the reduction of the model, $W$ is less unbalanced than $A$. 
The different degrees of accuracy for $N=0.13$ and $N=1 / 3$ can also be seen from the approximate error bounds (13) and (14). Because of the scaling performed by ZGGESX, they do not differ between the two different versions of QZ factorization. Yet, for the cluster of eigenvalues within the unit circle, the approximate error bound for the case $N=0.13$ is about $1.9 \times 10^{2}$ times larger in magnitude than for the case $N=1 / 3$. The error bound for the Schur decomposition is about $6.2 \times 10^{3}$ times larger.

Transformation and nonlinear solution. One way to overcome this problem is a reformulation of (26):

$$
\begin{aligned}
& 1=\frac{\left(C_{t}-\chi^{C} C_{t-1}\right)^{-\eta}}{\Lambda_{t}}, \\
& 1=v_{0} \frac{\left(N_{t}-\chi^{N} N_{t-1}\right)^{\nu_{1}}}{w_{t} \Lambda_{t}} .
\end{aligned}
$$

Indeed, we find negligible differences between the policy functions of the different solutions after this change. However, it may not always be obvious how to reformulate a model's equations or to transform its variables. Of course, one might be tempted to always resort to the nonlinear solution. Yet this requires additional programming, and inexperienced users of programming software might hesitate to take this step.

Balancing. However, there is a third possible solution: a previous balancing of the matrix pencil. Whereas the scaling performed by ZGGESX aims to make the elements of the scaled matrices $A$ and $B$ as close as possible to unity [see Ward (1981)], the scaling proposed by Lemonnier and van Dooren (2006) tries to make a matrix pencil as similar as possible to a pencil with orthogonal left and right eigenvectors. ${ }^{20}$ The algorithm of Lemonnier and Dooren (2006) computes two diagonal matrices $C$ and $D$ such that $A^{\prime}=C^{-1} A D$ and $B^{\prime}=C^{-1} B D$ represent the scaled pencil. We let ZGGESX factor this pencil, solved for the policy functions of the transformed problem, and transformed these back to those of the original problem. In this way we were able to reduce the maximum relative error between the QZ factorizations and the nonlinear solution to less than .6× $10^{-12}$. The success of this balancing scheme in reducing computational errors can also be seen from the error bound (13). In the case of $N=0.13$ the error for the eigenvalues within the first cluster drops from $0.22 \times 10^{-7}$ to $0.12 \times 10^{-12}$ for solution \#2 and from $0.20 \times 10^{-7}$ to $0.49 \times 10^{-13}$ for solution \# 3. For both solutions the Euler equation residual drops to $4.5 \%$.

\section{CONCLUSION}

The availability of easy-to-use toolkits for solving DSGE models has enhanced the widespread application of these models in macroeconomic research. The 
researcher supplies the equations of his model to programs such as DYNARE, which solve and simulate the model.

We demonstrate by means of an example that uninformed use of DSGE solution software may produce strange results. Researchers should be aware of those effects and take appropriate measures.

We consider a model that has been employed in studies of the equity premium puzzle. Because of matrices with very large and very small coefficients, the policy functions obtained from different ways of solving the model differ widely. The differences in policy functions give raise to differences in the second moments of model-simulated time series, so that the researcher may be misled with respect to the dynamic properties of the model. A very effective and particularly simple way to deal with this problem is the previous balancing of the matrices of the linearized system. The scheme that we employ differs from the scaling routinely undertaken by the procedures from the linear algebra package. Transformations of the model's variables and equations, as well as solving a system of nonlinear equations, are alternative, but less straightforward strategies for handling the problem.

\section{NOTES}

1. Others are the toolkit of Uhlig (1999), and the programs of Sims (2002).

2. See Heer and Maußner (2013) for an overview of those models.

3. The Online Appendix can be downloaded from http://www.wiwi.uni-augsburg.de/vwl/ maussner/pap/HKM_NA_Appendix.pdf.

4. Heer and Maußner (2009) present an illustrative example.

5. King and Watson $(1998,2002)$ present a different way to reduce a singular system of linear stochastic difference equations. The advantage of using the generalized Schur factorization, instead, is that it is implemented in the freely available LAPACK programs, and thus is easy to implement.

6. See, e.g., Golub and van Loan (1996, Theorem 7.7.1, p. 377), who also describe the algorithm to compute the factorization of $A$ and $B$. The set of all matrices of the form $B-\lambda A, \lambda \in \mathbf{C}$ is called a pencil. The eigenvalues of the pencil are the solutions of $|B-\lambda A|=0$. Unitary matrices $Q$ are complex-valued matrices whose conjugate (Hermitian) transpose equals the inverse of $Q$.

7. If $s_{i i}=0$ and $t_{i i} \neq 0$, the eigenvalue $\mu_{i i}=s_{i i} / t_{i i}$ of the pencil $|\mu A-B|=0$ is defined and equal to zero. Therefore, we can regard $\lambda_{i i}$ as an "infinite eigenvalue."

8. To see this, consider the last line of this system, which may be written

$$
\mathbf{E}_{t} \tilde{y}_{m, t+1}=\lambda_{m, m} \tilde{y}_{m, t}, \quad\left|\lambda_{m, m}\right|=\left|\left(t_{m, m} / s_{m, m}\right)\right|>1,
$$

where $s_{m, m}$ and $t_{m, m}$ with $m=n(w)+n(y)$ denote the last elements on the main diagonals of $S_{22}$ and $T_{22}$, respectively.

9. The routines are written in Fortran. $\mathrm{C}$ interfaces for these routines also exist.

10. The procedure of Heer and Maußner (2009) selects the eigenvalues according to $\left|\mu_{i}\right|<1$, $\mu_{i}=\alpha_{i} / \beta_{i}$.

11. See the LAPACK Users Guide on http://www.netlib.org/lapack/lug/node1.html.

12. Alternatively, following Heer and Maußner (2013), we could have chosen the unobserved parameters so that the model replicated certain empirical facts. However, because we use the model just as an example, the precise calibration does not matter.

13. $\Lambda_{t}$ denote the Lagrange multiplier of budget constraint (15).

14. See http://www.wiwi.uni-augsburg.de/vwl/maussner/lehrstuhl/maussner_en.html.

15. We report the steady state solution of the deterministic counterpart of the model in Table A.1 in the Online Appendix. 
16. See Aruoba et al. (2006) and Heer and Maußner (2008).

17. This equation is derived in the Online Appendix.

18. On a larger cube the policy functions occasionally return negative values, so that a meaningful comparison is not possible.

19. Note, however, that the Euler equation residuals inform about the error of an approximate solution, whereas Table 2 informs about different degrees of accuracy of the linear solution. It may still happen that numerically inaccurate coefficients in the linearized policy functions result in a better approximation. However, this is not the case here.

20. We are grateful to the referee who pointed us to this article.

\section{REFERENCES}

Aruoba, S. Boragan, Jesús Fernández-Villaverde, and Juan F. Rubio-Ramírez (2006) Comparing solution methods for dynamic equilibrium economies. Journal of Economic Dynamics and Control 30, 2477-2508.

Blanchard, Olivier J. and Charles M. Kahn (1980) The solution of linear difference models under rational expectations. Econometrica 48, 1305-1311.

De Paoli, Bianca, Alasdair Scott, and Olaf Weeken (2010) Asset pricing implications of a New Keynesian model. Journal of Economic Dynamics and Control 34, 2056-2073.

Golub, Gene H. and Charles F. Van Loan (1996) Matrix Computations, 3rd ed. Baltimore: Johns Hopkins University Press.

Hansen, Gary D. (1985) Indivisible labor and the business cycle. Journal of Monetary Economics 16, 309-327.

Heer, Burkhard and Alfred Maußner (2008) Computation of business cycle models: A comparison of numerical methods. Macroeconomic Dynamics 12, 641-663.

Heer, Burkhard and Alfred Maußner (2009) Computation of business-cycle models with the generalized Schur method. Indian Growth and Development Review 2, 173-182.

Heer, Burkhard and Alfred Maußner (2013) Asset returns, the business cycle, and the labor market. German Economic Review 14, 372-397.

Heiberger, Christopher, Torben Klarl, and Alfred Maußner (2015) On the uniqueness of solutions to rational expectations models. Economics Letters 128, 14-16.

Jermann, Urban J. (1998) Asset pricing in production economies. Journal of Monetary Economics 41, $257-275$.

Judd, Kenneth L. and Sy-Ming Guu (1997) Asymptotic methods for aggregate growth models. Journal of Economic Dynamics and Control 21, 1025-1042.

King, Robert G. and Mark W. Watson (1998) The solution of singular linear difference systems under rational expectations. International Economic Review 39, 1015-1026.

King, Robert G. and Mark W. Watson (2002) System reduction and solution algorithms for singular linear difference systems under rational expectations. Computational Economics 20, 57-86.

Klein, Paul (2000) Using the generalized Schur form to solve a multivariate linear rational expectations model. Journal of Economic Dynamics and Control 24, 1405-1423.

Lemonnier, Damien and Paul Van Dooren (2006) Balancing regular matrix pencils. SIAM Journal on Matrix Analysis and Applications 28(1), 253-263.

Schmitt-Grohé, Stephanie and Martin Uribe (2004) Solving dynamic general equilibrium models using a second-order approximation to the policy function. Journal of Economic Dynamics and Control 28, 755-775.

Sims, Christopher A. (2002) Solving linear rational expectations models. Computational Economics 20, 1-20.

Uhlig, Harald (1999) A toolkit for analysing nonlinear dynamic stochastic models easily. In Ramon Marimon and Andrew Scott (eds.), Computational Methods for the Study of Dynamic Economies. Oxford, UK: Oxford University Press.

Ward, Robert C. (1981) Balancing the generalized eigenvalue problem. SIAM Journal on Scientific and Statistical Computing 2(2), 141-152. 